\title{
Potential of the incidence angle effect on the radiometric calibration of full-waveform airborne laser scanning in urban areas
}

\author{
Fanar Mansour Abed \\ Surveying Engineering Department, College of Engineering, University of Baghdad, Baghdad, Iraq
}

Email address:

fanar_mansour@yahoo.com

\section{To cite this article:}

Fanar Mansour Abed, Potential of the Incidence Angle Effect on the Radiometric Calibration of Full-Waveform Airborne Laser Scanning in Urban Areas. American Journal of Remote Sensing. Vol. 1, No. 4, 2013, pp. 77-87. doi: 10.11648/j.ajrs.20130104.12

\begin{abstract}
Full-waveform airborne laser scanning has shown potential to better describe land cover features through the additional physical information it can provide alongside the standard geometric information. To fully utilize full-waveform for enhanced object recognition and feature extraction, it is essential to calibrate the backscattered energy of the received signal. The backscatter signal is affected by many variables during the travel between the sensor and the target. To eliminate these effects and deliver more reliable physical information for land cover features, the incidence angle effect was considered following the RSN method which was introduced in previous work. Following the radar equation, a comprehensive radiometric calibration routine was applied in this paper where the backscatter coefficient utilized to deliver the calibration constant. The calibrated results were achieved by means of backscatter cross-section and coefficient parameters in addition to the normalized parameters with respect to the incidence angle effect. The backscatter signals from overlapping flight lines were investigated and analyzed over various land cover types before and after calibration in the study site. Results show the potential of using the backscatter coefficient to deliver the calibration constant for the radiometric calibration purposes. It was also proven that the normalized backscatter coefficient with respect to incidence angle provides the greatest potential amongst the other backscatter parameters by delivering the optimal match between flight lines.
\end{abstract}

Keywords: Laser Scanning, Full-Waveform, Incidence Angle, Backscatter, Calibration, Analysis

\section{Introduction}

Airborne Laser Scanning (ALS) is a laser scanning system mounted on airborne platform and providing range measurements to the topographic Earth's surface. It delivers vertical accuracy of $5-10 \mathrm{~cm}$ and decimeter-level planimetric accuracy depending on the terrain type $[1,2]$. It usually uses to scan land cover features either from a fixed wing or a helicopter platform in order to collect the needed information to model the topographic surface [3]. ALS can be grouped into two main widely spread systems so called discrete-return and full-waveform (FWF) [4,2]. Both systems are designed to estimate range measurements using the physical concept of the pulsed laser mechanism. This acquired by measuring the round-trip time between the sensor and the ground target and back [5]. When the receiver only provides the start and the end of a signal at a certain rise time of the echo, then the system is called discrete-return. However, if the complete digital signal is digitized with extra information about the echo shape, then the system is called FWF.

FWF systems record the entire waveforms of the received signals which provide the possibility to deliver more description about surface features and more accurate range measurements [2]. In contrast with the discrete-return system, which provides end user with a single range measure to the ground target, FWF store the entire time history of the backscatter signal with a high resolution sampling [3]. With FWF the description of the land cover features can be improved by delivering geometric and physical characteristics of the surface objects by means of 3D point clouds and additional physical measurements. However this information is not recommended to be exploited for further analysis without a comprehensive calibration to increase the benefits of the collected positional and physical information [6]. Considering scanning geometry and terrain properties to improve the 
geometric quality of ALS data is essential [7], however the geometric calibration of ALS data is beyond the focus of this paper. Consequently, calibrating the backscatter energy towards fully utilizing FWF information is the overarching focus in this work.

The physical information from FWF-ALS data is affected by many variables during the travel between the sensor and the target. These include sensor properties, atmospheric conditions, incidence angle and other target characteristics effects [8, 9]. A robust radiometric calibration strategy must be capable of eliminating all aforementioned effects and delivering more reliable physical information for land cover features [10]. Multiple studies have concentrated on calibrating intensity values delivered from most of the ALS systems to improve the quality of the final products [11]. The intensity in the discrete systems is limited to represent the reflectance of the echo target. However, the physical additional information from FWF is representing all target characteristics including reflectance. Reference [8] presented two methods for intensity correction (data-driven and model-driven approaches). The data-driven approach is based on estimating a calibration parameter for the whole flight campaign by using the laser echo measurements delivered from multiple flying heights over a homogeneous extended area. The second approach is derived from the radar equation and is based on physical target properties of individual echoes and atmospheric conditions estimated from a single flying height. Both methods were found to successfully correct intensity values and are well-suited for implementation on large datasets. However, the second approach is more preferable as measurements are not required from multiple flying heights. Reference [12] discussed the potential of ALS intensity data in distinguishing land cover types and the capacity to be replaced with the spectral information delivered from the near-infrared band of optical images after intensity calibration. Reference [12] used ALTM 3070 ALS system with a $1064 \mathrm{~nm}$ wavelength. They examined the effects of reflectance and range on ALS intensity behavior using small-footprint data and investigated the radiometric properties on the delivered results. Their results show that intensity fails to differentiate vegetation from other objects. They determined that range exerts the greatest influence on intensity response over all land-cover types apart from vegetation. They concluded that this was because vegetation tends to generate a low intensity response, and was also mixed with the intensity of other targets. As a result, [12] recommend range radiometric calibration for all land-cover echoes except vegetation. Aiming to improve the quality of the derived products from ALS data, [6] present a combined calibration routine for ALS data to improve land-cover classification. They used a physical model based on the radar equation to calibrate the intensity data by taking sensor properties, topographic effects and atmospheric attenuation into consideration. After the implementation of this radiometric calibration, improvements in the classification results of up to $11.6 \%$ have delivered. However, the proposed angle of incidence correction approach of [6] is not robust enough for accurate applications. This is because the incidence angle estimation delivered from interpolated Surface (DSM) and not from the original point cloud, which incorporates more error source to the solution.

Reference [13] Introduce a practical calibration approach which uses portable artificial targets to calibrate ALS intensity data. Target reflectivity is measured by a spectra-radiometer from multiple incidence angles under laboratory conditions. Thereafter, laser intensity behavior at different wavelengths and incidence angles is modeled by calibrating the reflectivity measures against a reference panel [13]. This showed that surface brightness has a major effect on the backscattering energy at 1064 and $632.8 \mathrm{~nm}$ wavelengths. Reference [13] claimed that following this calibration routine the calibrated intensity data would facilitate separation between vegetation and other surface types supported improved classification and segmentation results. Reference [14] calibrates ALS intensity data from different flying heights using the same reference targets utilized in [13] approach based on radar equation. Their findings highlight the necessity for correcting ALS intensity values to account for differences in transmitted power, as well as for effects due to range, incidence angle, and atmospheric conditions. A similar approach was later presented by [15] but in this case using natural reference targets for the calibration process. However, despite this progress towards more effective calibration of ALS radiometric information, intensity data are not representative of all parameters which affect the received backscattered energy. In this sense, physical observables from FWF systems provide a superior, and more complete contribution.

Reference [16] introduces backscatter parameters which define all targets' properties, such as surface area, reflectivity, and the directionality of scattering, and discuss the potential of these parameters for calibration of the FWF backscatter signal. Subsequent investigations by [17] have delivered the development of this approach into a practical absolute radiometric calibration workflow. This approach utilizes a natural reference target of known backscatter characteristics to derive the calibration constant for the entire flight campaign. In contrast with previously proposed approaches, [17] recommend that the reflectivity of the reference target should be measured in the field, at the time of the survey, using a portable reflectometer to ensure that atmospheric conditions are consistent with those which exist during data acquisition. However, [17] ignore the effect of incidence angle on individual laser echoes during the calibration process. Building on these findings, [18] and later Alexander et al. (2010) investigate the potential of using the backscatter parameter to improve the classification of 3D point cloud data. Reference [18] was able to distinguish between broad canopy and terrain echoes by applying the backscatter cross-section parameter. 
Following this, the 3D point cloud was classified into vegetation and non-vegetation echoes with more than $90 \%$ overall mean accuracy. Following this, [19] detected improvements in classification performance through the backscatter cross-section parameter as compared with results delivered from utilizing the original echo amplitude signals. Later, [10] introduce a second parameter, the backscatter coefficient, which is a normalized version of the backscatter cross-section with respect to the area of illumination. The improved performance of the backscatter coefficient, over the backscatter cross-section for classification of 3D point clouds in FWF systems is subsequently demonstrated [10]. However, these studies fail to account for the effect of local incidence angle in the calibration process. Aiming to overcome this weakness, [20] incorporate the incidence angle effect in the radiometric calibration workflow proposed previously by [17]. Consequently, and by building upon the contribution of [19], [20] use the derived incidence angle to deliver the normalized backscatter coefficient for individual echoes. However, although, the presented routine is highly valid over planar features, they conclude that results are uncertain over more challenging rough surfaces and terrain.

In this paper, the potential of the angle of incidence effect on the radiometric calibration of FWF-ALS data is highlighted and analysed. The routine is based on the radar equation and relies on the robust incidence angle estimation using the developed RSN method introduced in a previous work. This includes the estimation of four different backscatter parameters for individual laser echoes and over different land cover targets in urban areas. The approach demonstrated the importance of the incidence angle inclusion in the radiometric calibration of FWF physical observables. This is through the fully utilising of FWF information for improved segmentation, classification and filtering techniques towards more accurate 3D object representation and modelling in urban sites.

\section{Test Data and Study Site}

This study utilizes small-footprint FWF-ALS data with a $1550 \mathrm{~nm}$ wavelength from Riegl LMS-Q560 scanner. The technical specifications of this system are described in the [21] data sheet. The test data was collected from the Bournemouth site located on the south coast of the UK and is composed of both man-made and natural land-cover features (Figure 1). The dataset has an average flying height of $350 \mathrm{~m}$ and was collected from a helicopter platform in May 2008. The dataset offers a high point density with more than 15 points $/ \mathrm{m} 2$ and a $0.18 \mathrm{~m}$ footprint diameter size. The scan campaign was flown in leaf-on condition with a simultaneous color photographs captured with on-board Hasselblad digital camera. The laser data acquisition and processing in addition to the raw imagery process to orthophotos was carry out by 3D Laser Mapping (3DLM) company with a ground sampling distance (GSD) of $0.05 \mathrm{~m}$. The dataset, together with the trajectory information and orthophoto coverage, were provided by Ordnance Survey, Great Britain's national mapping agency. The raw data was converted from waveforms to point cloud using RGD post processing method [22]. This processing has delivered the laser point cloud in the scanner's coordinate system. Later, the Riegl commercial software (RiWrold) was used to convert the points to the WGS84 coordinate system to be converted finally to the UK national grid system through TerraScan commercial software.

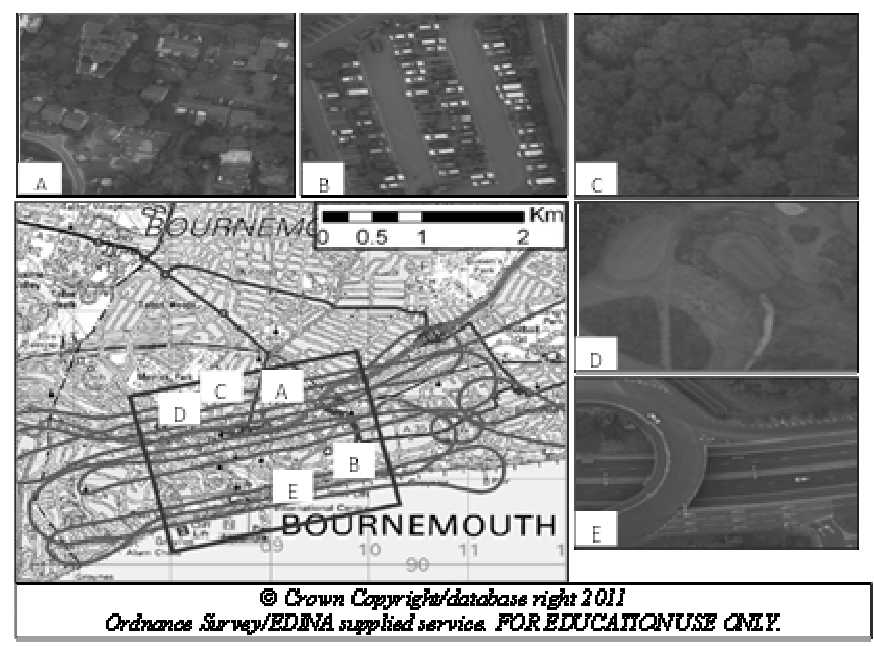

Figure 1. Study site, with red polygon defining the ground coverage and trajectory depicted in grey. Sample land cover features are detailed orthophotos A to E, and described further in Section 4.

\section{Methodology}

\subsection{Theory}

Following the radar equation, laser backscatter cross-section $\sigma$ is a measure of directional scattering power that defines all target characteristics including scattering direction, reflectivity and area of illumination [23]. In FWF-ALS system and when using the Gaussian function to retrieve the laser signal such as in the case of RGD method, the received power can be represented as the product of echo amplitude $\hat{P}_{i}$ and echo width $s_{p, i}$ and noted as $\widehat{P}_{i} s_{p, i}$ for simplification [24]. Assuming that all unknown in the radar equation are combined in one single constant, the calibration constant $\left(C_{c a l}\right)$ can be delivered for a certain ALS campaign [17]:

To estimate the calibration constant, it is required to have a reference target with known reflectivity to deliver $\sigma$ value (refer to $[17,25]$ ). $A_{i}$ can be estimated from the range $R$, the beam divergence $\beta_{t}$, and angle of incidence $\alpha$ as in 1 .

$$
A_{i}=\frac{R^{2} \beta_{t}^{2} \pi}{4 \cos \alpha_{i}}
$$

Once $C$ cal is delivered, $\sigma$ can be estimated for individual echoes of the entire dataset [26]. However, the backscatter coefficient $(\gamma)$ is more stable than $\sigma$ as the later tends to vary with different system and target characteristics [18]. 
When the incidence angle of the laser beam is changed, then the illumination area is also changed therefore $\gamma$ can be called the normalized backscatter cross-section to the illumination area which can be derived as follows:

$$
\gamma=\frac{\sigma}{A_{i} \cos \alpha}
$$

However, neither $\sigma$ nor $\gamma$ are free from the incidence angle effect [27]. Therefore both of the two parameters should be normalized with respect to the incidence angle effect by dividing each parameter with the cosine of the incidence angle within the Lambertian scatterer assumption as illustrated in 3 and 4 respectively.

$$
\begin{aligned}
\sigma_{\alpha} & =\frac{\sigma}{\cos \alpha} \\
\gamma_{\alpha} & =\frac{\gamma}{\cos \alpha}
\end{aligned}
$$

\subsection{Calibration Routine}

A practical and reliable radiometric calibration routine, which accounts for all the variables affecting the backscattered energy, is presented herein including the essential factor of angle of incidence. The routine is based on the radar equation and relies on a robust incidence angle estimation method so called the RSN, refer to [9]. The complete workflow of the developed radiometric calibration routine considering all these factors is illustrated in Figure 2.

Firstly, the FWF dataset has been post-processed using the RGD software [22]. Following this, geometric and physical information is delivered for individual FWF echoes. In order to calibrate the dataset, the four aforementioned backscatter parameters should be estimated for individual laser echoes. To achieve this, incidence angle estimation for individual echoes has to be first undertaken.

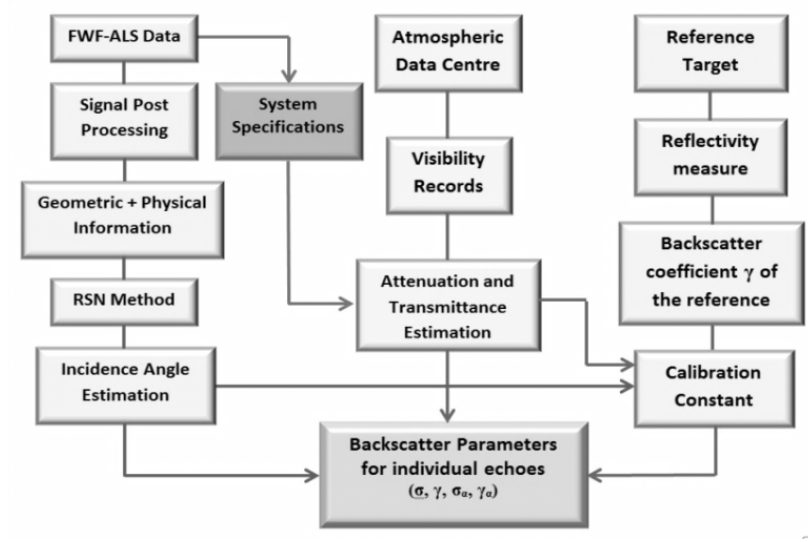

Figure 2. Flowchart of the developed radiometric calibration routine.

As incidence angle is a function of illumination direction from the sensor to the target and the surface normal vector associated with the point, a Robust Surface Normal (RSN) method has been implemented [9]. This aims to produce a robust surface normal estimation over any land cover type and thus deliver more reliable incidence angle estimation for individual FWF echoes. The method is based on the $k$-nearest neighbor's algorithm by including a minimum number of points to estimate the normal following 3D moment invariant theory. It defines the 3D spherical volume by including only the three nearest Euclidian neighboring points to the point in question. FWF echo amplitude values are used as a weighting function in the invariant definition. To ensure robustness of the delivered normal, a threshold value, based on data density and point accuracy, is applied to the vector dot product in 3D space. This determines whether the selected points provide a suitable basis for accepting the 3D volume definition, or whether the current neighborhood system should be rejected and a new definition computed, (refer to [9] for details). The RSN method is based on robust planarity checking that delivers a highly accurate normal value over planar surfaces and the best approximate normal over non-planar surfaces. This is opposed to an unreliable normal delivered from fitting a plane to a group of points with different orientations which is commonly acquired over natural land coverage and surfaces with rough or challenging trends of small details. This is mainly because the normal delivered from available methods could be reliable for some of the points used to define the orientation of the surface but not necessary for the point of interest itself which would translate to an erroneous normalized echo amplitude value. A robust normal estimation method is essential for different laser scanning applications, including the segmentation of point clouds.

In order to undertake the radiometric calibration, firstly the RSN method was applied to the reference targets echoes to guarantee the consideration of the incidence angle effect on the reference target backscattered energy. In order to deliver a comprehensive calibrated backscatter signal, the energy loss due to atmospheric scattering and absorption effects during time of flight was considered in the calibration model. The atmospheric transmittance was estimated based on the model described by [8] and presented in 5 .

$$
\eta_{\text {atm }}=10^{-2 H a / 2000}
$$

Where $\eta_{\text {ntm }}$ is the atmospheric transmittance, $H$ is the flying height in meters, and $a$ is the atmospheric attenuation in $\mathrm{dB} / \mathrm{Km}$.

The atmospheric attenuation coefficient of the laser power is strongly affected by laser wavelength and visibility records which was delivered from the nearest metrological station. The attenuation modeling was undertaken using the model described by [28] as shown in 6.

$$
a=\frac{3.91}{V}\left(\frac{\lambda}{550}\right)^{-a}
$$


Where $V$ is the visibility measured in $\mathrm{km}, \lambda$ is the wavelength in $\mathrm{nm}$, and $q$ is the size distribution of the scattering particles.

In order to deliver the calibration constant for the scan campaign, a reference target with known reflectivity is required. Twenty-two 1m-radius circular PVC targets were distributed in the study site and therefore utilized as reference for the absolute calibration routine. Although successive reflectivity measures should be undertaken for the reference targets during data capture to guarantee the same surface conditions, this condition could not met in this dataset. This is because the radiometric calibration was not the intended function when these targets were firstly deployed in the field. Therefore, post-survey indoor reflectivity measurements was undertaken to deliver the backscatter parameters of the reference target. The indoor reflectivity measurements were run using an Analytical Spectral Device (ASD) [29]. The measurements were conducted in a dark room using a portable lamp mounted on a fixed tripod for illumination purposes. Successive measurements were undertaken using $1^{\circ}$ and $8^{\circ}$ field of view optics over multiple regions of the reference target to assure homogeneity. For each lens, five different sets of incidence angle measurements were undertaken at $0^{\circ}, 10^{\circ}$, $20^{\circ}, 30^{\circ}$, and $40^{\circ}$ of successive 25 measurements per angle using white reference and raw modes, refer to Alasdair [30, 31] for further details about measurements modes. Thereafter, post processing of the recorded spectral data was undertaken. This include comparing the recorded data with the ASD white-reference reflectivity (Spectralon ${ }^{\circledR}$ panel), followed by mean value computations for individual lenses to deliver the best absolute reflectivity estimation for the reference target from different incidence angle settings.

As the backscatter coefficient $(\gamma)$ has a close relationship with bi-conical reflectance [10], it has been considered as the parameter of choice to estimate the calibration constant in this research. An ideal Lambertian scatter has been assumed in the case of reference targets, with the incidence angle effect considered in the reflectivity computations following 7 .

$$
\rho=\rho_{0} \cos (\alpha)
$$

Where $\rho$ is the reflectivity when assuming an ideal Lambertian scatterer, $\rho_{o}$ is the reflectivity at incidence angle zero, and $\alpha$ is the incidence angle.

Calibration constants were then delivered for all reference target echoes based on the backscatter coefficient values. This means that both the incidence angle and atmospheric effects have been considered over reference targets echoes. To avoid noise effects, a mean calibration constant was then determined. The calibration constant was subsequently utilized for the determination of the four backscatter parameters $\left(\sigma, \gamma, \sigma_{\alpha}, \gamma_{\alpha}\right)$ for individual echoes.

The methodology proposed here aims to calibrate FWF backscatter signals by eliminating the discrepancies between signals delivered from overlapping flight lines. To achieve this goal, it was proposed to find the backscatter parameter that delivers the best match between signals from overlapping flight lines after calibration. In order to assess which configuration is able to best eliminate flight line discrepancies, the four backscatter parameters $\left(\sigma, \gamma, \sigma_{\alpha}, \gamma_{\alpha}\right)$ were investigated for individual echoes within the selected regions in the tested dataset. The $\sigma_{\alpha}$ and $\gamma_{\alpha}$ parameters demonstrate the influence of the incidence angle effect on the reflected backscatter signal which translate the performance of the RSN method. Firstly, the signals from overlapping flight lines were investigated individually to check whether they follow the distribution of the normal curve. This is essential as the majority of the standard statistical tests are reliant on the normal distribution assumption. To check the normality of the individual flight line signals, normality statistical test was implemented. Based on the normality check results, the decision is made in whether to utilize the F-test or Levene's statistical test when testing variances of the datasets from the overlapping flight lines. The F-test is adopted when the tested datasets are found to be normally distributed while Levene's test should be considered if the normality test delivers questionable distribution results, (refer to [32] for details about the statistical tests). Thereafter, statistical and visual analysis of performance is implemented to check the means of the comparative signals.

\section{Results and Discussion}

\subsection{Reference Target Reflectivity Measure}

Figure 3 shows a sample of spectral reflectivity measurements recorded by $1^{\circ}$ fore-optic and $0^{\circ}$ incidence angle over the PVC reference target. The $\mathrm{X}$-axis represents wavelength spectral range while the Y-axis shows the absolute reflectivity values after post processing. The graph shows the distribution of the successive 25 series measurements, with the first series always eliminated in order to assure high measurement precision. This is because the first measurement could deliver an unstable value due to effects with the previous measurement. As the atmosphere strongly absorbs radiation between 1350 and $1460 \mathrm{~nm}$ wavelengths, and also between 1790 and $1960 \mathrm{~nm}$, these bands were removed as it can be realized from the data gaps in Figure 3. This is because these spectral regions are very sensitive to the atmosphere and thus very noisy data is delivered in these two regions.

It can be realized from the graph that at the $1550 \mathrm{~nm}$ wavelength which defines the wavelength of the Riegl LMS-Q560 system, the absolute reflectivity for the PVC target is shown to be approximately $70 \%$ at nadir. This was delivered from all measurements after accounting for the multiple incidence angle settings effect. Therefore, a 0.7 reflectivity value for the reference target was adopted for further processing in the current dataset. 


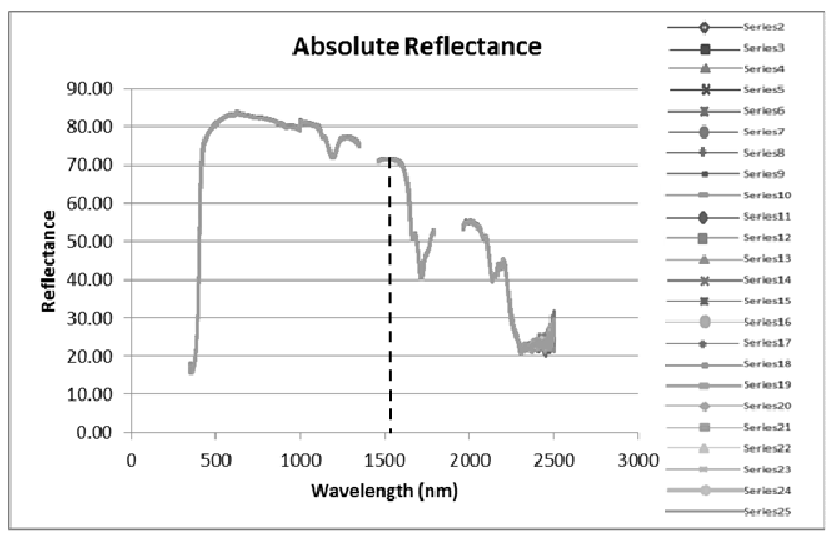

Figure 3. Absolute reflectivity measurements of a sample series records over the $P V C$ reference target using $1^{\circ}$ fore-optic and $0^{\circ}$ incidence angle.

\subsection{Calibration Constant Assessment}

In this research, after delivering the calibration constant for the tested dataset, it is necessary to assess the potential of this constant by compare it to constants from other approaches. [19] Presented an alternative routine to estimate the calibration constant for the same dataset. They estimated the calibration constant by utilizing natural target (asphalt road sections) as reference to calibrate the whole dataset through the adoption of $\sigma$ value to deliver the backscatter energy of the reference target. They utilized a literature reflectance value of 0.25 for the asphalt, and no reflectivity measurements were undertaken in order to calibrate this dataset. The incidence angle effect was neglected throughout [19] routine and instead only near-nadir echoes were used. This is in contrast with the approach presented here, which uses $\gamma$ as a measure of the backscatter energy for the PVC reference target.

To assess the calibration constant presented in this research, the routine is compared with that presented by [19] over a road target which appears in overlapping flight lines in the tested dataset. This target was selected in order to minimize the incidence angle effect as much as possible in order to allow more direct comparison to [19] method. To assure approximately similar conditions (e.g. range and scan angle) from both flight lines, the road target was selected to meet the geometry proposed in Figure 4. Thus in perfect conditions, the received backscatter signals from overlapping flight lines are assumed to be the same over this particular target.

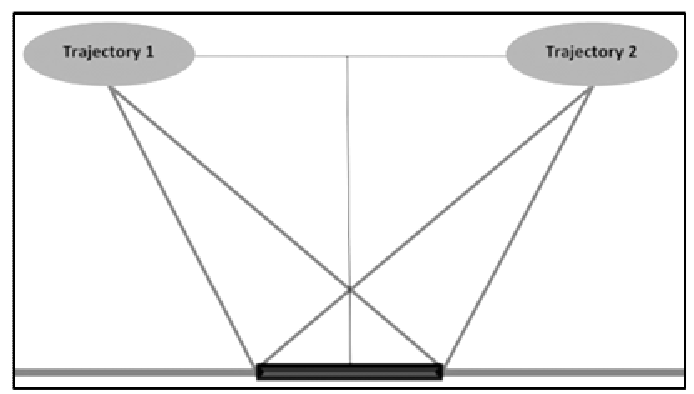

Figure 4. The geometry of the road target selected to check the potential of using $P V C$ as a reference target.
Firstly, the presented technique was applied on the individual road target echoes and the signals compared from both overlapping flight lines as shown in Figure 5. The histogram of the $\gamma$ parameter in Figure 5-c shows a significant improvement over the original amplitude signals in Figure 5-a whereas the results from the $\sigma$ parameter (Figure 5-b) shows a significant drawback. This was approved by the standard deviation and mean differences in percentage between signals from overlapping flight lines as illustrated in Figure 5. The drawback in the $\sigma$ results was not expected which might be acquired by overcorrecting or differences in target characteristics between flight lines. However, the $\gamma$ parameter shows encouraging results as the differences were reduced significantly in a comparison with the original signals.

Further, the results of $\gamma_{\alpha}$ deliver a nearly perfect match between the overlapping flight lines signals as highlighted in Figure 5-e and demonstrated through small mean and standard deviation differences. Consequently $\sigma_{\alpha}$ outcomes in Figure 5-d shows reduction in the mean and standard deviation differences as compared with Figure 5-b results. Although the investigated target was selected to be as flat as possible, it seems that it is not a perfectly flat surface and the incidence angle is still affecting the received signals as demonstrated by Figure 5-d and Figure 5-e. It is clear that the results of $\gamma_{\alpha}$ agreed with the assumption made when selecting this target in first place which assures similar conditions (e.g. range and scan angle) from both flight lines. Consequently, new $\gamma$ signals were estimated for individual echoes enclosed within the selected target from overlapping flight lines using the calibration constant estimated by [19]. The histogram results of $\gamma$ following [19] routine are demonstrated in Figure 5-f. As the approach of [19] neglects the incidence angle effect throughout the calibration workflow, no incidence angle is available to use for comparison. Therefore the comparison between the presentenced and [19] routines is based on $\gamma$ results of the road target delivered from both routines.

It can be realized from Figure 5-f that [19] routine proves to deliver significant improvements after calibration as compared with the original signals in Figure 5-a. This can be justified by delivering low mean and standard deviation differences between overlapping flight lines in a comparison with the original signals from Figure 5-a. However, the potential of the results improvements delivered from both routines is still need further investigations. To achieve this, a statistical 2-sample T-test is used to compare $\gamma$ results delivered from both approaches. This statistical test is designed to compare the means of two sample datasets based on variance check analysis, by delivering an index value known as the P-value that better describes the probability of both datasets are being significantly different to each other or not, (refer to [32] for further details). In this case, the datasets used in this comparison are representing the signals from overlapping flight lines obtained through the two approaches. The test was applied using the $95 \%$ confidence level. Thus, a P-value of less than $5 \%$ means that 
the datasets are significantly different. However, this test cannot be applied without checking the variance of both

datasets.

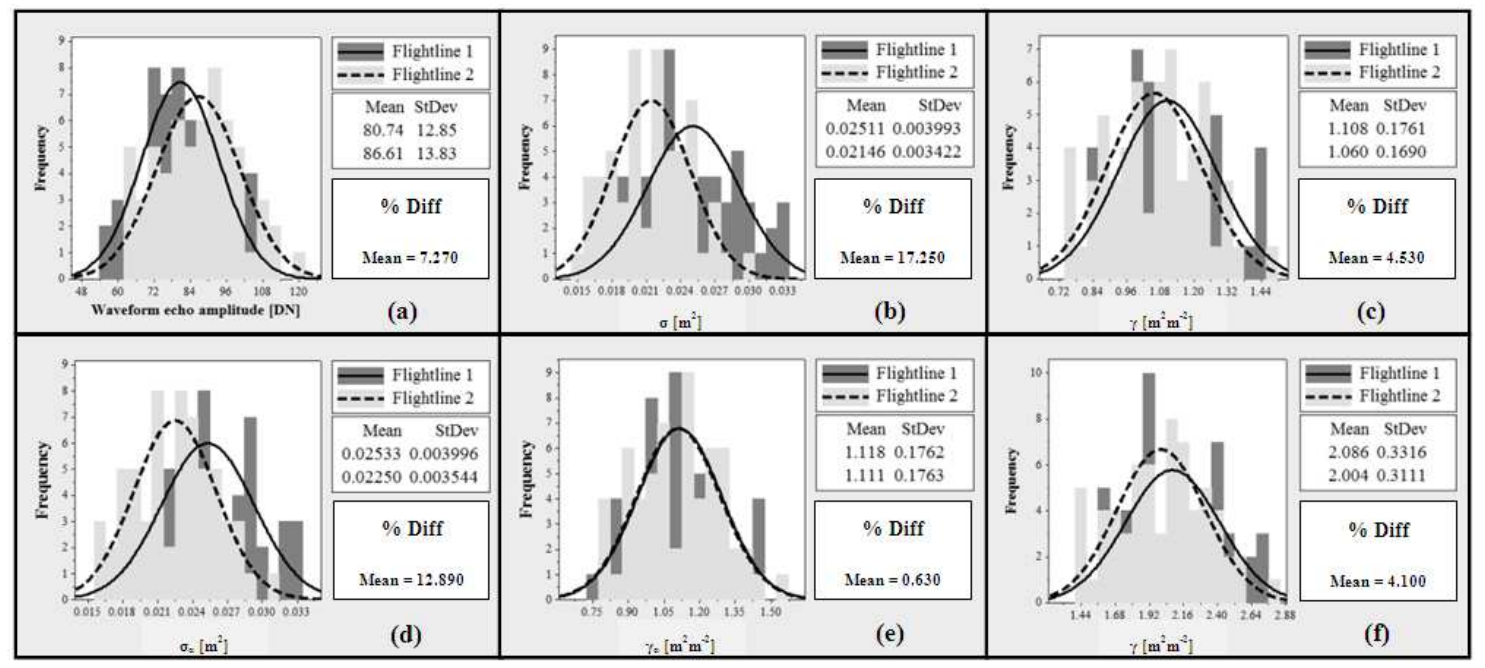

Figure 5. The histogram and the statistical reports of the backscatter signals before and after calibration of a selected road target from overlapping flight lines: (a) the original amplitude signals; (b)-(e) the four backscatter parameters $\left(\sigma, \gamma, \sigma_{\sigma}, \gamma_{\alpha}\right)$ respectively after calibration with the presented routine; $(f)$ the $\gamma$ signals delivered from following [19] routine.

This was undertaken using the F-test, which compares the variances and checks whether the datasets have similar variances or not, in order to use the outcome as an input to the subsequent T-test. Figure 6 demonstrates the F-test results which obtained from comparing the variances of the backscatter coefficient signals delivered from overlapping flight lines through both approaches. As it can be seen, the $\mathrm{P}$-value delivered from the presented routine is higher than the corresponding value delivered from following [19] routine. This is also evidenced from the steeper gradient of the dashed line connecting between the mean values from the overlapping flight lines signals delivered from [19] routine than the line connecting between the signals from the presented routine. These outcomes are visualized from Figure 6 through the inclination angles delivered from both approaches.

Table 1 illustrates the T-test statistical reports for both approaches. It shows that the estimate for the differences between the means is lying within the confidence interval (CI) in both approaches. Consequently, it can be concluded that the datasets in both approaches are not significantly different. This is also clear from the P-value results which proved that no significant differences can be observed through both approaches. That means, both approaches deliver a significant agreement between signals from overlapping flight lines after calibration. However, the developed approach delivers a higher P-value than the one delivered from [19] approach. This is also evidenced through the lower T-value delivered from the presented routine where T-value is interpreted opposite the P-value (low T-value means better match). This confirms the potential of using $\gamma$ rather than $\sigma$ to estimate the backscatter energy of the reference target and delivering the calibration constant for radiometric calibration purposes. This is because when adopting $\gamma$ parameter the incidence angle variation over the reference target echoes is considered which shows potential to change the illumination area and therefore affecting the calibration constant value. Although considering incidence angle effect for individual echoes after calibration through $\gamma_{\alpha}$ parameter can deliver more comprehensive comparison evidence which proves to deliver the best agreements between overlapping flight lines, this could not be applied here as [19] discard this essential effect.

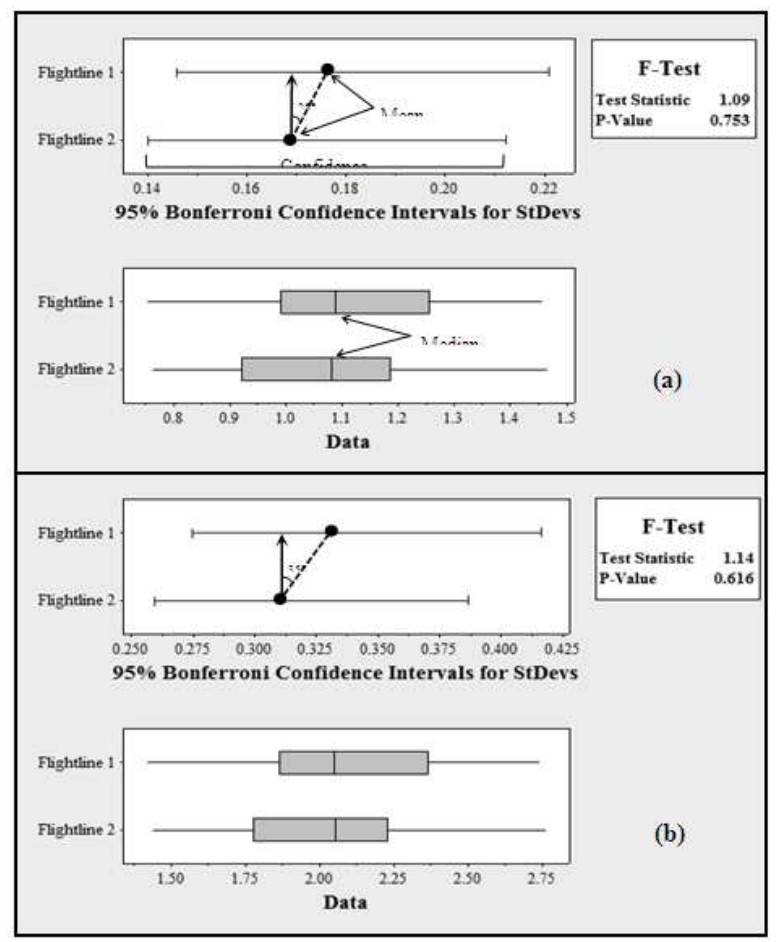

Figure 6. F-test of the backscatter coefficient signals from overlapping flight lines over a selected road target in the study site: (a) results from the presented calibration routine; (b) results from applying [19] routine. 
Table 1. Two-sample T-test reports of $\gamma$ signals delivered from overlapping flight lines through both comparative approaches.

\begin{tabular}{ll}
\hline & Difference = mean (Flight line 1) - mean (Flight line 2) \\
The presented & Estimate for difference: 0.0476 \\
routine & $95 \%$ CI for difference: $\quad(-0.0148,0.1099)$ \\
& T-Test of difference: $\quad$ T-Value $=1.35, \quad$ P-Value $=0.299$ \\
\hline & Difference = mean (Flight line 1) - mean (Flight line 2) \\
& Estimate for difference: 0.0823 \\
& $95 \%$ CI for difference: $\quad(-0.0315,0.1961)$ \\
& T-Test of difference: $\quad$ T-Value $=1.43, \quad$ P-Value $=0.155$ \\
\hline
\end{tabular}

\subsection{Assessing the Calibrated Backscatter Signal}

To assess the calibrated backscatter signals of individual echoes, an interest area $(\approx 100 \mathrm{~m} \times 60 \mathrm{~m})$ in the urban site of the study campaign was analyzed and investigated. The area is extended over a good variety of land cover features with different surface properties (refer to Figure 8-a) were the signals have been analyzed and compared from overlapping flight lines. In order to assess which configuration is able to best eliminate flight line discrepancies, the four aforementioned backscatter parameters were considered.

To summarize these outcomes over the selected interest area by means of statistical conception, a Pareto chart of all backscatter parameters is produced in Figure 7. The chart describes the discrepancies between flight lines delivered from the four backscatter parameters based on the T-values following the T-test. The results show a visible reduction in the backscattered signals through $\gamma_{\alpha}$ parameter which show to deliver the smallest differences between overlapping flight line signals in comparison with other parameters. Figure 7 proves that the $\gamma_{\alpha}$ parameter delivers the best match through the lowest T-value (highest P-values), while $\sigma$ shows the poorest performance of all the parameters. This is because the backscatter coefficient parameter normalized with respect to incidence angle, can better account for the differences in signals acquired from trajectories of different position and orientation.

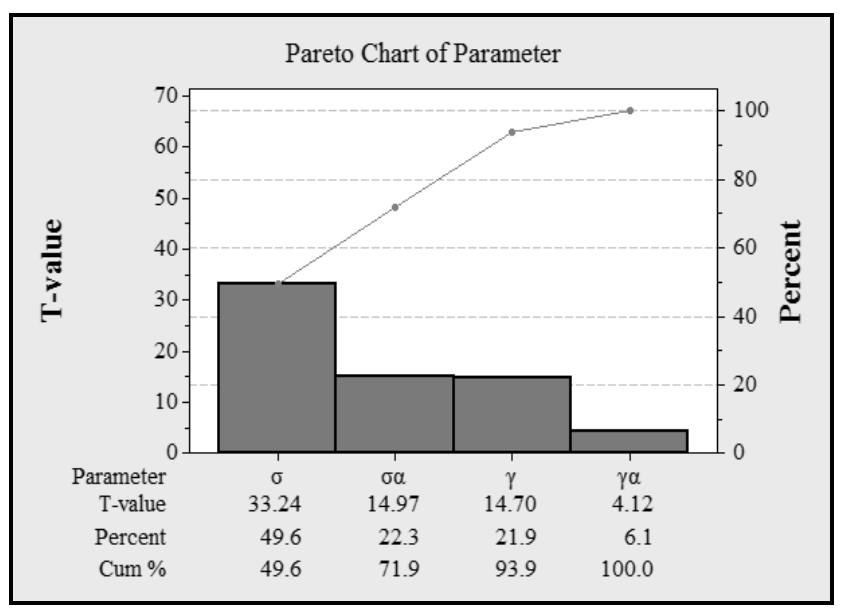

Figure 7. Assessing the calibrated backscatter signals: (a) orthophoto of the selected interest area; (b) pareto chart of the backscatter parameters over the interest area in the study site.
In order to demonstrate the configuration which is best eliminate flight line discrepancies, the coefficient of variation of the four backscatter parameters were estimated based on the standard deviation and mean values delivered for individual flight lines. Thereafter, the difference between the overlapping flight lines was delivered for individual backscatter parameters by means of coefficient of variation as shown in Table 2.

Table 2. Coefficient of variation statistics of the backscatter parameters from the overlapping flight lines before and after calibration over the interest area.

\begin{tabular}{llllll}
\hline CofVar & Amplitude & $\boldsymbol{\sigma}$ & $\boldsymbol{\gamma}$ & $\boldsymbol{\sigma}_{\boldsymbol{\alpha}}$ & $\boldsymbol{\gamma}_{\boldsymbol{\alpha}}$ \\
\hline Flight line 1 & 0.525 & 0.551 & 0.536 & 0.518 & 0.513 \\
Flight line 2 & 0.638 & 0.624 & 0.572 & 0.562 & 0.526 \\
Difference & 0.113 & 0.073 & 0.036 & 0.044 & 0.013 \\
\hline
\end{tabular}

It is evidenced from Table 2 that $\gamma \alpha$ parameter is delivering the smallest coefficient of variation differences between the overlapping flight lines in a comparison with the rest of the backscatter parameters after calibration. It proves the potential of the presented calibration technique through the $\gamma_{\alpha}$ parameter which shows a clear reduction in the signals between flight lines after calibration. Finally and in order to visualize the performance of the calibration routine through the $\gamma_{\alpha}$ parameter, scale unified difference maps for overlapping flight line signals were produced as shown in Figure 8. These include the original amplitude signals and their corresponding calibrated signals delivered from the $\gamma_{\alpha}$ parameter. The reduction in the signal differences is clearly visualized over roofs, roads and grass regions in Figure 8, demonstrating the minimization of differences between the backscatter signals before and after calibration. It is also evident that a relative improvement in the signal differences has been detected over vegetated regions in this case.

Seeking to deliver a comprehensive and qualitative conclusion about the optimal backscatter configuration for individual feature targets, Twenty-six variant targets were tested and analyzed within the study site. Each target was selected to represent feature with homogeneous characteristics. These targets were grouped into seven different categories and demonstrated in Table 3 by means of coefficient of variation statistical differences between the backscatter signals from overlapping flight lines signals before and after calibration. These differences represent the mean value of the coefficient of variation between flight lines of all targets included within individual category based on the standard deviation and mean values.

The results delivered from Table 3 indicate marked improvements after calibration with all backscatter parameters in a comparison with the original amplitude differences. However, the $\gamma_{\alpha}$ parameter delivers the optimal match between flight lines except over tree category, where the $\sigma$ parameter shows better performance. These outcomes 
highlight the potential of the incidence angle over all tested targets except over trees because of the unreliable estimation of the incidence angles over tree echoes which evidenced from bad results of the $\sigma_{\alpha}$ and $\gamma_{\alpha}$ in this particular category. However, $\gamma_{\alpha}$ parameter outcomes prove the optimal agreement between overlapping flight line signals over all other categories including the low mowed grass and hedges which was evidenced by the small value of the coefficient of variation differences between flight lines as demonstrated in Table 3.
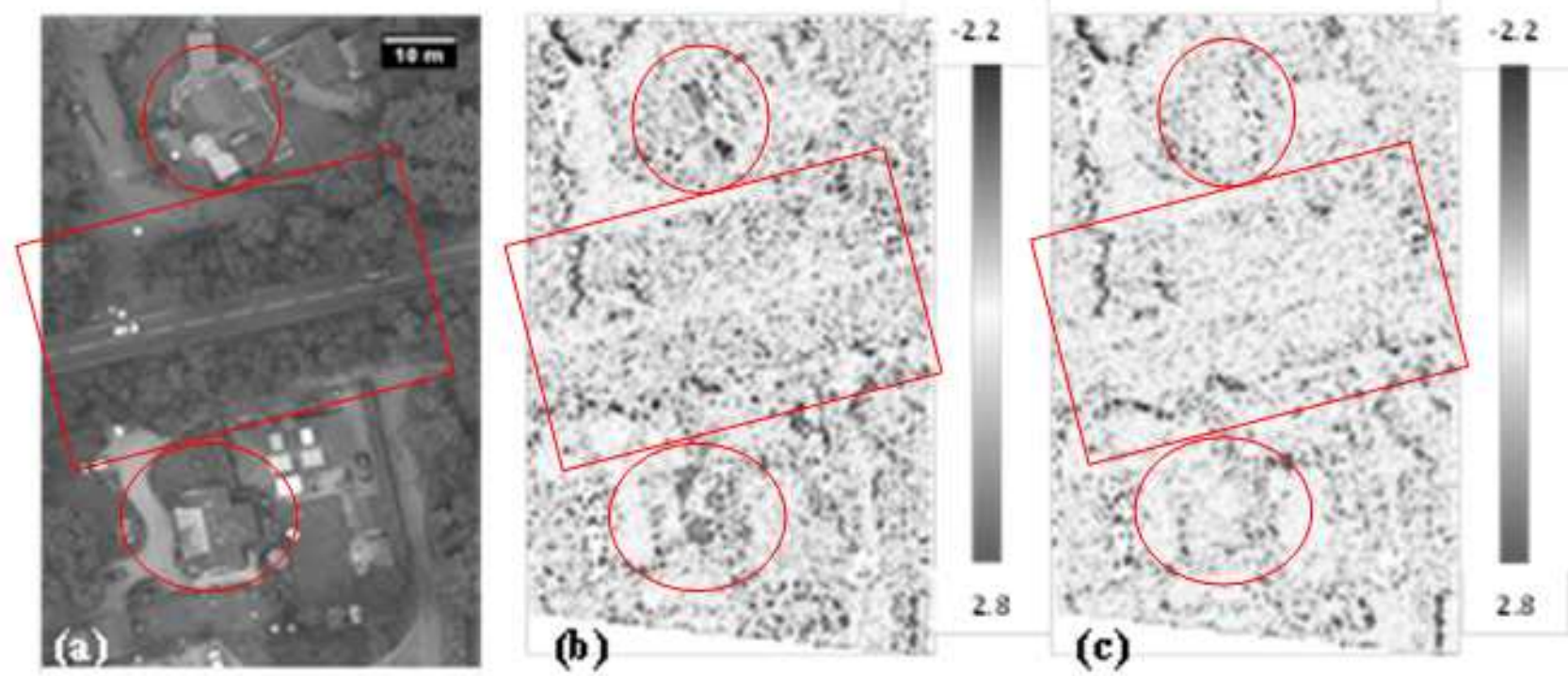

Figure 8. Scale unified difference maps from two overlapping flight lines for the interest area: (a) orthophoto; (b) original echo amplitude signals; (c) $\gamma_{\alpha}$ signals.

Table 3. Coefficient of variation statistics of the backscatter parameters from the overlapping flight lines before and after calibration of different land covers categories in the tested site.

\begin{tabular}{|c|c|c|c|c|c|c|c|c|}
\hline \multirow{2}{*}{ Category } & \multirow{2}{*}{$\begin{array}{l}\text { No. of } \\
\text { targets }\end{array}$} & \multicolumn{2}{|l|}{ No. of points } & \multicolumn{5}{|c|}{ Mean difference of CofVar } \\
\hline & & Flight line 1 & Flight line 2 & Amplitude & $\sigma$ & $\gamma$ & $\sigma_{\alpha}$ & $\gamma_{\alpha}$ \\
\hline Artificial ground & 5 & 6260 & 5825 & 0.032 & 0.016 & 0.011 & 0.015 & 0.007 \\
\hline House roof & 6 & 6434 & 6279 & 0.045 & 0.043 & 0.022 & 0.027 & 0.011 \\
\hline Car & 5 & 513 & 538 & 0.087 & 0.066 & 0.018 & 0.033 & 0.008 \\
\hline Low mowed grass & 3 & 4391 & 4407 & 0.044 & 0.041 & 0.027 & 0.034 & 0.024 \\
\hline Hedge & 3 & 6613 & 6489 & 0.051 & 0.023 & 0.020 & 0.023 & 0.005 \\
\hline Tree & 4 & 12930 & 14279 & 0.030 & 0.019 & 0.036 & 0.265 & 0.274 \\
\hline
\end{tabular}

\section{Conclusion}

This paper presents a robust strategy for radiometric calibration of full-waveform ALS data. It aims to consider all variables affecting the backscatter signal in the calibration routine in order to deliver more appropriate signal for further applications. This was achieved by considering the signal variation acquired due to different target characteristics through incidence angle considerations which significantly affect the illumination area. Therefore considering the normalized backscatter cross-section according to the illumination area $(\gamma)$ to deliver the backscatter energy of the reference target echoes is highly recommended when applying radiometric calibration. This was proven through the adoption of this parameter to estimate the calibration constant for the scan campaign and also evidenced through the calibrated signals for individual echoes. Consequently, the incidence angle shows potential on the calibration routine which should be considered through the adoption of $\gamma_{\alpha}$ backscatter parameter to reduce the discrepancies between signals from overlapping flight lines. This was achieved through the adoption of a robust method to estimate the orientation of the land cover features with various characteristics. The results prove the robustness of the RSN method to estimate the incidence angle for individual echoes which is applicable to a wide variety of land cover types and dataset point densities.

In conclusion, the presented radiometric calibration routine show the effective role of the incidence angle parameter in reducing backscatter signal discrepancies 
between overlapping flight lines after calibration. It was proved that using reference target with known reflectivity value delivered from practical measurements can lead to a better match between calibrated backscatter signals from overlapping flight lines than using published literature value. However, it is recommended to perform the reflectivity measurements of the reference target at time of data capture in order to deliver the same conditions of the day of scan. Finally, results show the potential of using $\gamma$ rather than $\sigma$ to estimate the calibration constant for radiometric calibration purposes. Consequently, it was also proven that the $\gamma_{\alpha}$ parameter provides the greatest potential amongst the four investigated backscatter parameters $(\sigma, \gamma$, $\sigma_{\alpha,} \gamma_{\alpha}$ ) by delivering the optimal match between flight lines except over vegetation where $\sigma$ shows better outcomes.

\section{Acknowledgements}

The author would like to acknowledge Ordnance Survey for royalty-free provision of data used in this research. The author would also like to acknowledge Geomatic Engineering staff members within the school of Civil Engineering and Geosciences in Newcastle University in the UK for their advice, guidance, help, and support throughout the accomplishment of this research.

\section{References}

[1] F. Ackermann, (1999), 'Airborne laser scanning- present status and future expectations', ISPRS Journal of Photogrammetry and Remote Sensing, 54 (2-3), pp. 64-67.

[2] C. Mallet, and F. Bretar, (2009), 'Full-waveform topographic lidar: State-of-the-art', ISPRS Journal of Photogrammetry and Remote Sensing, 64 (1), 16 p.

[3] G. Vosselman, and H.-G. Maas, (2010), Airborne and terrestrial laser scanning. Scotland, UK: Whittles Publishing

[4] J. Shan, and C. K. Toth, (2009), Topographic laser ranging and scanning - principles and processing. FL, USA: Taylor \& Francis.

[5] W. Wagner, A. Ullrich, T. Melzer, C. Briese, and K. Kraus, (2004), 'From single-pulse to full-waveform airborne laser scanners: potential and practical chalenges', International Archives of Photogrammetry, Remote Sensing and Spatial Information Sciences, 35 (B3), pp. 201-206.

[6] A. Habib, A. Kersting, A. Shaker, and W. Y. Yan, (2011), 'Geometric calibration and radiometric correction of lidar data and their impact on the quality of derived products', Sensors, 11 (9), pp. 9069-9097.

[7] A. Habib, A., Kersting, K. Bang, and D.-C. Lee, (2010), 'Alternative methodologies for the internal quality control of parallel lidar strips', IEEE Transactions on Geoscience and Remote Sensing, 48 (1), pp. 221-236.

[8] B. Höfle, and N. Pfeifer, (2007), 'Correction of laser scanning intensity data: Data and model-driven approaches', ISPRS Journal of Photogrammetry and Remote Sensing, 62 (6), pp. 415-433.
[9] F. Abed, J. Mills, and P. Miller, (2012), 'Echo amplitude normalisation of full-waveform airborne laser scanning data based on robust incidence angle estimation', IEEE Transactions on Geoscience and Remote Sensing, 50 (7), pp. 2910-2918.

[10] W. Wagner, (2010), 'Radiometric calibration of small-footprint full-waveform airborne laser scanner measurements: Basic physical concepts', ISPRS Journal of Photogrammetry and Remote Sensing, 65 (6), pp. 505-513.

[11] F. Abed, (2012), 'Calibration of full-waveform aerial laser scanning data for 3D object segmentation', PhD thesis, Newcastle University, Newcastle, UK.

[12] J.-S. Yoon, J.-I. Shin, and K.-S. Lee, (2008), 'Land cover characteristics of airborne lidar intensity data: A case study', IEEE Geoscience and Remote Sensing Letters, 5 (4), pp. 801-805.

[13] S. Kaasalainen, E. Ahokas, J. Hyyppa, and J. Suomalainen, (2005), 'Study of surface brightness from backscatter laser intensity: calibration of laser data', IEEE Geoscience and Remote Sensing Letters, 2 (3), pp. 255-259.

[14] E. Ahokas, S. Kaasalainen, J. Hyyppa, and J. Suomalainen, (2006), 'Calibration of the Optech ALTM 3100 laser scanner intensity data using brightness targets', International Archives of Photogrammetry, Remote Sensing and Spatial Information Sciences, 36 (A1), 6 p.

[15] F. Coren, and P. Sterzai, (2006), 'Radiometric correction in laser scanning', International Journal of Remote Sensing, 27 (15), pp. 3097-3104.

[16] W. Wagner, J.Hyyppa, A. Ullrich, H. Lehner, C. Briese, and S. Kaasalainen, (2008a), 'Radiometric calibration of full-waveform small-footprint airborne laser scanners', International Archives of Photogrammetry, Remote Sensing and Spatial Information Sciences, 37 (B1), pp. 163-168.

[17] C. Briese, B. Höfle, H. Lehner, W. Wagner, M. Pfennigbauer, and A. Ullrich, (2008), 'Calibration of full-waveform airborne laser scanning data for object classification', In: Proceedings of SPIE 2008. pp. 69501-69508, DOI: 10.1117/12.781086.

[18] W. Wagner, M. Hollaus, C. Briese, and V. Ducic, (2008b), '3D vegetation mapping using small-footprint full-waveform airborne laser scanners', International Archives of Photogrammetry, Remote Sensing and Spatial Information Sciences, 29 (5), pp. 1433-1452.

[19] C. Alexander, K. Tansey, J. Kaduk, D. Holland, and N. J. Tate (2010), 'Backscatter coeefficient as an attribute for the classification of full-waveform airborne laser scanning data in urban areas', ISPRS Journal of Photogrammetry and Remote Sensing, 65 (5), pp. 423-432.

[20] H. Lehner, and C. Briese, (2010), 'Radiometric calibration of Full-Waveform airborne laser scanning data based on natural surfaces', International Archives of Photogrammetry, Remote Sensing and Spatial Information Sciences, 38 (7B), pp. 360-365.

[21] Riegl. (2009), 'LMS-Q560', Airborne Laser Scanner for full-waveform analysis, [Online]. Available at: http://www.riegl.com/uploads/tx_pxpriegldownloads/10 Da taSheet_Q560_20-09-2010_01.pdf (Accessed: 20 Sep 2010).

[22] Y.-C Lin, J. Mills, and S. Smith-Voysey, (2010), 'Rigorous 
pulse detection from full-waveform airborne laser scanning data', International Journal of Remote Sensing, 31 (5), pp. $1303-1324$

[23] A. V. Jelalian, (1992), Laser radar systems. Boston, United Kingdom: Artech House.

[24] W. Wagner, A. Ullrich, V. Ducic, T. Melzer, and N. Studnicka, (2006), 'Gaussian decomposition and calibration of a novel small-footprint full-waveform digitising airborne laser scanner', ISPRS Journal of Photogrammetry and Remote Sensing, 60 (2), pp. 100-112.

[25] B. Höfle, M. Hollaus, and J. Hagenauer, (2012), 'Urban vegetation detection using radiometrically calibrated small-footprint full-waveform airborne lidar data', ISPRS Journal of Photogrammetry \& Remote Sensing, 67 (1), pp. 134-147.

[26] H. Lehner, H. Kager, A. Roncat, and A. Zlinszky, (2011), 'Consideration of laser pulse fluctuations and automatic gain control in radiometric calibration of airborne laser scanning data', In: Proceedings of The 6th ISPRS Student Consortium and Working Group VI/5 Summer School. Fayetteville, NC, USA, ISPRS WG VI/5: On CD-ROM.
[27] G. Mandlburger, N. Pfeifer, C. Ressl, C. Briese, A. Roncat, H. Lehner, and W. Mucke, (2010), 'Algorithms and tools for airborne lidar data processing from a scientific perspective', In: Proceedings of The European Lidar Mapping Forum (ELMF) 2010. Hague/ Netherlands : On CD-ROM.

[28] I. Kim, B. McArthur, and K. Eric, (2000), 'Comparison of laser beam propagation at $785 \mathrm{~nm}$ and $1550 \mathrm{~nm}$ in fog and haze for optical wireless communications', In: Proceedings of SPIE-Optical wireless communications III. pp. 26-37.

[29] NERC (2009), NERC Field Spectroscopy Facility. Available at: http://fsf.nerc.ac.uk/ (Accessed: 10 Oct 2009).

[30] M. A. Alasdair, (2007a), Field guid for the ASD FieldSpec Pro - raw DN mode. Available at: http://fsf.nerc.ac.uk/resources/guides/pdf_guides/asd_guide_ v2 dn.pdf (Accessed: 15 Oct 2009).

[31] M. A. Alasdair, (2007b), Field guid for the ASD FieldSpec Pro - white reference mode. Available at: http://fsf.nerc.ac.uk/resources/guides/pdf guides/asd guide v2_wr.pdf (Accessed: 15 Oct 2009).

[32] D. C. Montgomery, (2005), Design and analysis of experiments. Chichester, UK: Willy-BlackWell. 